mothers of preterm infants to offer support in the postpartum period.

\section{DIFFERENCES IN POSTPARTUM POLICY AFTER MATERNAL USE OF SELECTIVE SEROTONIN-REUPTAKE INHIBITORS DURING PREGNANCY}

doi:10.1136/archdischild-2012-302724.1292

AJE Wakker-Deelen, AVN Schmetz, AC de Mol. Department of Pediatrics, Albert Schweitzer Hospital, Dordrecht, The Netherlands

Background and Aim $\pm 10 \%$ of women has a depression during pregnancy and selective serotonin reuptake inhibitors (SSRI) are frequently used. After the use of SSRI's approximately $30 \%$ of neonates show adverse effects. We studied different policies in the Netherlands in term neonates after maternal SSRI use and findings of standard 48-hour monitor observation and glucose testing.

Methods A questionnaire about local policy in neonates after maternal SSRI use was performed in all Dutch hospitals. Next to this we describe the occurrence of incidents and hypoglycemia in a 5.5-years cohort of term neonates $(\mathrm{n}=138)$, in which standard monitor observation and glucose testing was performed.

Results The questionnaire response rate was $79 \%$. Standard observation is conducted in $96 \%$ of the hospitals, $77 \%$ on the maternity ward and $23 \%$ on the neonatology ward, using a monitor. The majority ( $\mathrm{n}=53,73 \%$ ) observes neonates for 48 hours (range 12-72 hours). Standard glucose testing is performed in $12 \%$ of hospitals. Ambulatory follow-up is performed in $30 \%$ of hospitals. Our cohort study showed that if no incidents occurred during the first 24 hours of observation, no incidents will occur thereafter. Glucoses were below cut off value in $12 \%$ mainly at 1 hour after birth, resolving with oral feeding.

Conclusions There are many differences in postnatal care for neonates born after maternal SSRI use. Based on our cohort study it seems unnecessary to prolong monitor observation after 24 hours if no incidents occurred. Standard glucose testing should not be performed.

\section{UMBILICAL ARTERY INTIMA-MEDIA AND WALL THICKNESS IN INFANTS OF DIABETIC MOTHERS AND ITS RELATION TO MATERNAL HYPERGLYCEMIA}

doi:10.1136/archdischild-2012-302724.1293

'YU Sarikabadayi, , 10 Aydemir, 'HG Kanmaz, ${ }^{1} \mathrm{C}$ Aydemir, ${ }^{1} \mathrm{SS}$ Oguz, ' 0 Erdeve, ${ }^{2} \mathrm{EG}$ Yapar Eyi, ${ }^{3}$ S Zergeroglu, 'U Dilmen. 'Department of Neonatology, Zekai Tahir Burak Maternity Teaching Hospital; ' 2 Department of Perinatology, Zekai Tahir Burak Maternity Teaching Hospital; ${ }^{3}$ Department of Pathology, Zekai Tahir Burak Maternity Teaching Hospital, Ankara, Turkey

Background Children who are large for gestational age at birth and exposed to an intrauterine environment of either diabetes or maternal obesity are at increased risk of developing metabolic syndrome. This can be explained by exposure to high glucose and insulin levels in utero causing altered fetal adaptation and changes in normal fetal programming.

Objectives The aim of the study was to evaluate preclinical atherosclerosis begins in utero.

Methods We measured the umbilical artery wall thickness (ruWT) in the third trimester by obstetric ultrasound and umbilical artery intima media thickness (UIMT) in pathologic specimens of the umbilical cords obtained shortly after delivery and we investigated the relation between the these measurements and serum insulin, c-peptide level in cord blood and homeostasis model assessment of insulin resistance (HOMA-IR) in infants of diabetic mother (IDM). Study group divided into two groups as; large for gestational age (LGA)/IDM group, appropriate for gestational age (AGA)/IDM group and compared with control group.
Results The LGA/IDM group had significantly higher insulin $(p<0.001)$, c-peptides $(p=0.018)$ and HOMA-IR levels $(p<0.001)$ compared to AGA/IDM group and controls. LGA/IDM group had significantly higher ruWT $(p=0.013)$ and $\operatorname{UIMT}(\mathrm{p}<0.001)$ values compared to AGA/IDM group and controls. LGA/IDM group has increased umbilical artery intima-media and wall thickness which correlates with severity of maternal hyperglycemia.

Conclusions Measurement of ruWT in third trimester is feasible, reproducible and strongly correlated with pathological measurements.

\section{IS MATERNAL DEPRESSIVE SYMPTOMATOLOGY EFFECTIVE ON EXCLUSIVE BREASTFEEDING DURING POSTPARTUM 6 WEEKS?}

doi:10.1136/archdischild-2012-302724.1294

'A Annagür, ${ }^{2 B}$ Burçak Annagür, ${ }^{3} \mathrm{~A}$ Şahin, ${ }^{3 R}$ Örs, ${ }^{4} \mathrm{~F}$ Kara. ${ }^{1}$ Selcuk University, Selcuklu Medical Faculty, Department of Neonatology; ' ${ }^{S}$ elcuk University, Selcuklu Medical Faculty; ${ }^{3}$ Konya University, Meram Medical Faculty, Department of Neonatology: ${ }^{4}$ Selcuk University, Selcuklu Medical Faculty, Department of Public Health, Konya, Turkey

Aim The aim of this prospective study was to examine the relationship between exclusive breastfeeding and postpartum depressive symptomatology. Our hypothesis was that mothers with depressive symptoms initially, fail exclusive breastfeeding.

Methods One hundred ninety seven mothers attended the study. The participants were interviewed two times. The first visit was within the first 48 hours after birth. The Edinburgh Postnatal Depression Scale (EPDS) was completed by the participants. The second interview performed 6 weeks. Participants answered methods of breastfeeding for 6 weeks, any methodological problems and nipple pain. EPDS was completed by the participants in 6 weeks. Newborns were term infant.

Results All the participants divided into two groups as exclusive breastfeeding and mix-feeding (partial breastfeeding and/or bottle feeding). Both groups were compared in terms of features, such as mode of delivery, parity, prevalence of depressive symptomatology (at 48-h and $6 \mathrm{wk}$ ) and delayed onset of lactation within first 48. Statistical significance was found for only two variables which delayed onset of lactation within first 48-h and gestational age.

Discussion Contrary to our expectation, effect of higher maternal depressive symptomatology was not demonstrated on exclusive breastfeeding during 6 weeks after delivery. However, the important finding of this study that delayed onset of nutrition within the first 48 hours affects negatively exclusive breastfeeding during 6 weeks after delivery.

Conclusion Clinicians especially should pay attention that lactation difficulty during the first week postpartum. Early lactation difficulties are associated with greater risk of early termination of breastfeeding and lower breastfeeding success.

\section{CORD BLOOD THEOPHYLLINE LEVELS AND RESPIRATORY MORBIDITIES IN PRETERM INFANTS}

doi:10.1136/archdischild-2012-302724.1295

TH Çelik, A Korkmaz, Ş Yiğit, M Yurdakök. Department of Pediatrics, Division of Neonatology, Hacettepe University, Faculty of Medicine, Ankara, Turkey

Background Methylxantines such as caffeine and its metabolites, which are taken by pregnant women via daily foods, are completely transferred to the fetus through placenta. Caffeine has been shown to improve respiratory functions and decrease bronchopulmonary dysplasia in preterm infants. However respiratory effects of prenatal caffeine exposure are not known completely in newborn infants.

Aim We aimed to investigate whether there is a relationship between cord blood theopylline levels (which is a main caffeine 